\title{
CO-DESIGNING SOCIAL SIMULATION MODELS FOR POLICY ADVISE: LESSONS LEARNED FROM THE INFSO-SKIN STUDY
}

\author{
Petra Ahrweiler \\ Demian Frank \\ TISSS Lab \\ Johannes Gutenberg University \\ Jakob-Welder-Weg 20 \\ 55128 Mainz. Germany \\ Petra.ahrweiler@uni-mainz.de
}

\author{
Nigel Gilbert \\ Centre for Research on \\ Social Simulation \\ University of Surrey \\ Guildford GU2 7XH, UK \\ n.gilbert@surrey.ac.uk
}

\begin{abstract}
This paper describes a participatory approach to co-designing social simulation models with policymakers using a case study of modeling European Commission policy. Managing the collaboration of a wide range of individuals or organizations is challenging but increasingly important as policy making becomes more complex. A framework for a co-design process based on a participatory approach is proposed. The framework suggests that the collaborative design should go through the following phases: Identifying user questions, data provision, model discussion for validation, visualization of results and discussing scope and limitations with stakeholders. Key findings are that the co-design process requires communication skills, patience, willingness to compromise, and motivation to make the formal world of modelers and the narrative world of policymaking meet. Furthermore, especially in participatory modeling scenarios, stakeholders have to be included not only in the provision of data, but also in order to confirm the existence, quality and availability of data.
\end{abstract}

Keywords: co-design, policy modeling, participatory process.

\section{INTRODUCTION}

Participative approaches to research and innovation are becoming more and more common, because they reflect the networked modes of these activities referenced by concepts such as "Mode 2" (cf. Gibbons et al. 1994), "Triple Helix" (cf. Etzkowitz and Leydesdorff 1997) and "Innovation Networks" (cf. Ahrweiler 2010). However, they vary across disciplines in focus and process. 
For example in 'Community-based Participatory Research' stemming from development studies, ideas of democratically inspired partnership and societal welfare are central: "Community-based participatory research is an orientation to research that emphasizes 'equitable' engagement of partners throughout the research process, from problem definition, through data collection and analysis, to dissemination and use of findings to help effect change" (Cacari-Stone et al. 2015: 1615). In management science, the approach of value co-creation is more focused on business opportunities: "Co-creation is joint creation and evolution of value with stakeholding individuals, intensified and enacted through platforms of engagement, virtualized and emergent from ecosystems of capabilities, and actualized and embodied in domains of experiences, expanding wealth-welfare-wellbeing" (Ramaswamy and Ozcan 2014). In computer science, "technology co-design" combines software development and the theory of sociotechnical systems: it suggests "that technologies and work practices are best co-designed using participatory methods in the workplace setting, drawing on such common-sense guiding principles as staff being able to access and control the resources they need to do their jobs and insisting that processes should be minimally specified to support adaptive local solutions" (Greenhalgh et al. 2016: 406).

In computational simulation studies, the idea of "Participatory Modeling" extends to two different activities: in the first, it describes an "action that engages the implicit and explicit knowledge of stakeholders to create formalized and shared representation(s) of reality. In this process, the participants co-formulate the problem and use modeling practices to aid in the description, solution, and decisionmaking actions of the group (...) engaging non-scientists in the scientific process. The participants structure the problem, describe the system, create a computer model of the system, use the model to test policy interventions, and propose one or more solutions" (Wikipedia, accessed 1 March 2019). In the second, "Participatory Modeling" describes an activity where a model is created consisting of both human and artificial components, e.g. human and computational agents, which interact with each other for the purpose of the model (e.g. interactive crowdsourcing: cf. Lasecki and Bigham 2013). It is the first meaning of the term "Participatory Modeling" that will be the concern of this paper.

Involving stakeholders in policy and management modeling activities has been extensively applied in socio-ecological management (Jones et al. 2009, Mendoza and Prabhu 2006, Robles-Morua et al. 2014, van Eeten et al. 2002), although including stakeholder perspectives and expertise can increase the complexity of models. An example of doing so is the "Companion Modeling" framework (Barreteau et al. 2003, Etienne 2013), which involves object formation, problem definition, discussion, and elaboration. However, though this approach might be the one of choice for complex multi-stakeholder contexts such as natural resource management (Bouamrane et al. 2016, Berthet et al. 2016), where heterogeneous interests need to be negotiated in conflict-prone discussion arenas with high uncertainty requiring many loops, de-briefings and societal reflections, it does not seem to be easily adaptable to the area of public policy modeling (Gilbert et al. 2018).

Although policy modeling for policy advice shares the complexity of the target (Geyer and Cairney 2015), it is also a straightforward service activity with policymakers and policy analysts as clients of modelers who are often contracted under demanding budget and time restrictions. High utility of results and efficiency of the process are mandatory requirements of any policy advice project using computational models (cf. Aodha and Edmonds 2017). This requires a lean and systematic process among modelers and policymakers with fewer and different features compared to the sophisticated framework of a Companion Modeling project (cf. Figure 2.2. in Barreteau et al. 2013: 24). The task is to develop appropriate support to help stakeholders and policy actors engage with and benefit from those with modeling and assessment expertise by establishing a set of resources to help both sides negotiate the relationship (cf. Jager and Edmonds 2015).

In the following, a comparatively lean participatory approach for the special work relationship between modelers and policy clients will be suggested. 


\section{THE CASE STUDY}

Developing a co-design process in participatory modeling using an agent-based model (ABM) implies work-intensive mechanisms for both the modelers and the stakeholders. In this section of the paper, these mechanisms will be outlined using as a case study an example of our work with the Simulating Knowledge dynamics in Innovation Networks (SKIN) model in its application to research policy modeling. There are now a number of policy modeling studies using SKIN (Gilbert et al. 2014) for stakeholder-intensive policy modeling. To illustrate the co-design scenario, which enabled the inductive development of a more systematic and generalizable participatory modeling process, we will here refer to just one recent example, on the impact assessment and ex-ante evaluation of European funding policies in the Information and Communication Technologies (ICT) research domain (Ahrweiler et al. 2015).

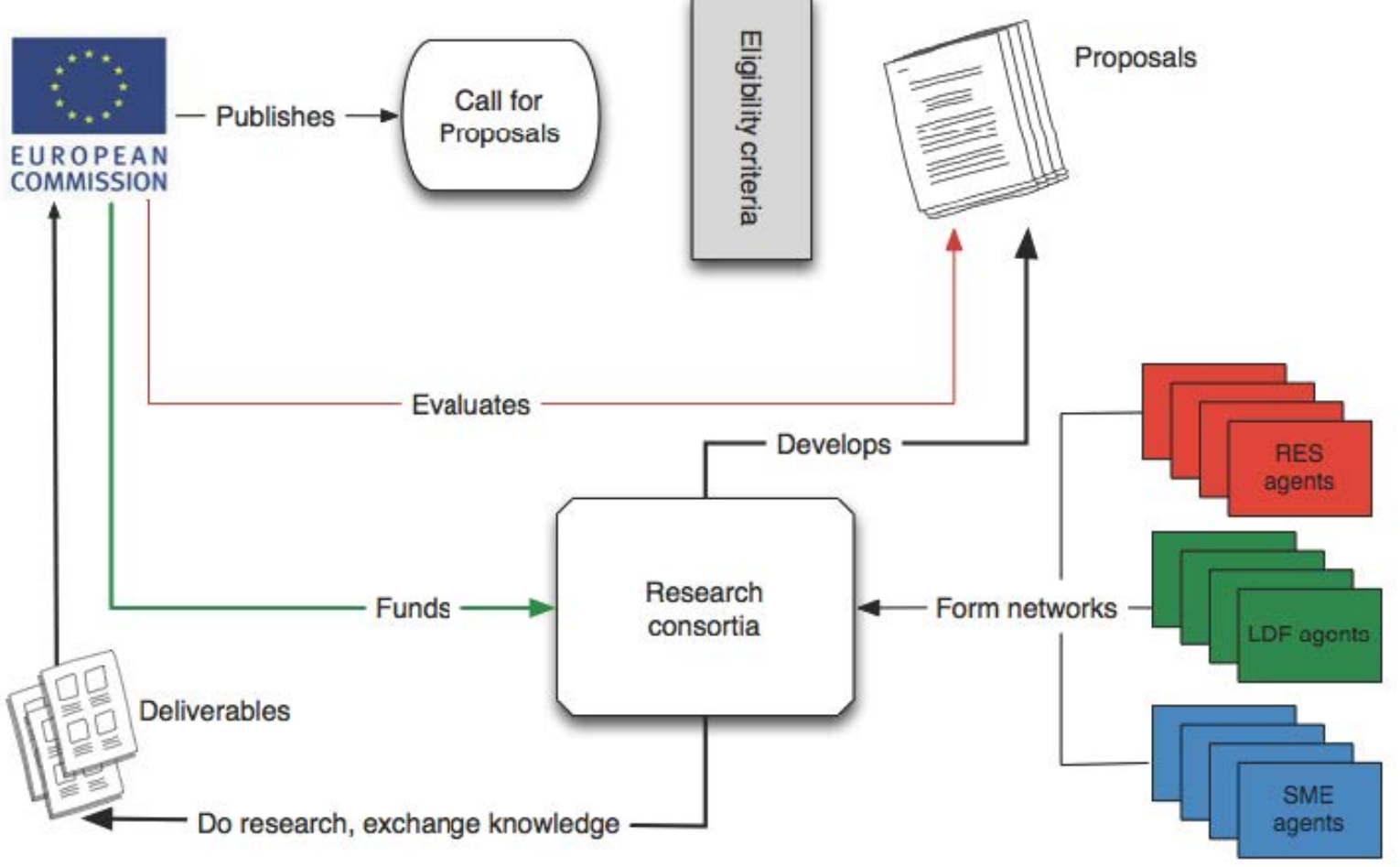

Figure 1: Flowchart of INFSO-SKIN.

The INFSO-SKIN application, developed for the Directorate General Information Society and Media of the European Commission (DG INFSO), was intended to help to understand and manage the relationship between research funding and the goals of EU research policy. The agents of the INFSO-SKIN application are research institutions such as universities, large diversified firms and small and mediumsized enterprises (SMEs). The model (see Figure 1) simulated real-world activity in which the Calls of the Commission specify the composition of consortia, the minimum number of partners, and the length of the project; the deadline for submission; a range of capabilities, a sufficient number of which must appear in an eligible proposal; and the number of projects that will be funded. The rules of interaction and decision implemented in the model corresponded to Framework Programme (FP) rules; to increase the usefulness for policy designers, the names of the rules corresponded closely to Framework Programme terminology. 
For the Calls 1-6 that had occurred in FP7, the model used empirical information on the number of participants and the number of funded projects, together with data on project size (as measured by participant numbers), duration and average funding. Analysis of this information produced data on the functioning of, and relationships within, actual collaboration networks within the context of the Framework Programme. Using this data to calibrate the model provided a good match with the empirical data from EU-funded ICT networks in FP7: the model accurately reflected what actually happened and could be used as a test bed for potential policy choices.

Altering elements of the model that equate to policy interventions such as the amount of funding, the size of consortia, or encouraging specific sections of the research community, enabled the use of INFSOSKIN as a tool for modeling and evaluating the results of specific interactions between policies, funding strategies and agents. Because changing parameters within the model is analogous to applying different policy options in the real world, the model could be used to examine the likely real-world effects of different policy options before they were implemented.

The first contact with "the real world" occurred in the definition phase of the project. What do the stakeholders want to know in terms of policies for a certain research or innovation network? Identifying relevant issues, discussing interesting aspects about them, forming questions and suggesting hypotheses for potential answers was a first important step. It aimed to conclude with a finite set of questions and concrete designs for the experiments that are to be addressed by the model. This was an interactive and participative process between the study team, which knew about the possibilities and limitations of the model, and the stakeholders, who could be assumed to know the relevant issues in their day-to-day practice of policymaking. After discussing the evaluative questions for the ex-ante evaluation part of this study with the stakeholders from DG INFSO, the following questions were singled out for experiments:

1. What if there are no changes, and funding policies of DG INFSO continued in Horizon 2020 as they were in FP7?

2. What if there are changes to the currently eight thematic areas funded in the ICT domain prioritizing certain areas in Horizon 2020 ?

3. What if there are changes to the instruments of funding and larger/smaller consortia in Horizon 2020 than in FP7 are funded?

4. What if there are interventions concerning the scope or outreach of funding, providing much more / much less resource to more / fewer actors?

5. What if there are interventions concerning the participation of certain actors in the network (e.g. SMEs)?

The next step (see Figure 2) was to collect relevant data to address these questions and hypotheses. The issues were not different from the ones every empirical researcher is confronted with. To identify relevant variables for operationalizing hypotheses, to be as simple as possible but as detailed as necessary for description and explanation, is in line with the requirements of all empirical social research. For SKIN, the most important data are about knowledge dynamics (e.g. knowledge flows, amount of knowledge, and diversity of knowledge) and their indicators (e.g. publications, patents, and innovative ideas), and about the dynamics of actors, networks, their measures, and their performance (e.g. descriptive statistics about actors, network analysis measures, and aggregate performance data). 


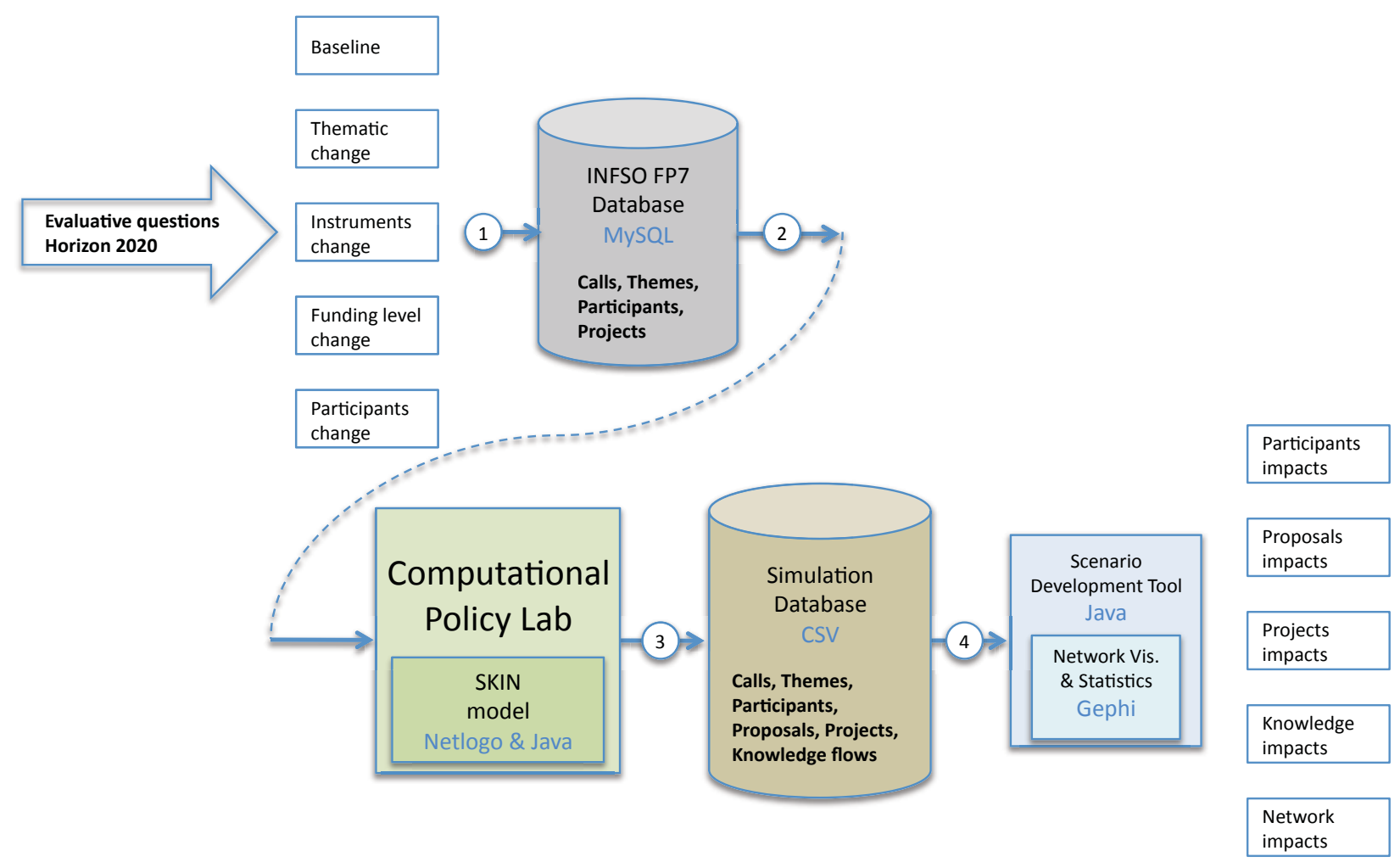

Figure 2: Horizon 2020 study workflow (Schilperoord and Ahrweiler 2014). First (on the left), a set of issues was isolated, in discussion with stakeholders. Data describing the network of FP7 projects and participants, by theme and Call, obtained from DG INFSO were entered into a database. These data were used to calibrate the INFSO-SKIN model. This model was then used to generate simulated data under various policy options. The simulated data were fed into a second database and visualized using additional network visualization and statistical software in order to assess the expected impacts of those policy options.

These data were used to calibrate the initial knowledge bases of the agents, the social configurations of agents ("starting networks"), and the configuration of an environment at a given point in time. DG INFSO provided the data needed to calibrate the knowledge bases of the agents (in this case the research organizations in the European research area), the descriptive statistics on agents and networks and their interactions (in this case data on funded organizations and projects in ICT under FP7).

The time series data were used to validate the simulations by comparing the empirical data with the simulation outputs. Once we were satisfied with the model performance in that respect, experiments were conducted and the artificially-produced data analyzed and interpreted. The stakeholders were again invited to provide their feedback and suggestions about how to fine-tune and adapt the study to their changing user requirements as the study proceeded.

The last step was again stakeholder-centered as it involved visualization and communication of data and results. We had to prove the credibility of the work and the commitment of the stakeholders to the policy modeling activity.

We worked from an already existing application of the SKIN model adapted to the European research area (Scholz et al. 2010), implemented the scenarios according to the evaluative questions and produced artificial data as output of the simulations. The results were reported in a Final Report that was presented to the European Cabinet and were communicated to the stakeholders at DG INFSO. 
Ahrweiler, Gilbert and Frank

\section{THE PROCESS}

From experience with this case study, we started developing a systematic process for participatory modeling using the agent-based SKIN model, which we could test against a range of other applications of this model (Ahrweiler et al. 2016). Roughly, this process can be divided into five building blocks, which we introduce again referring to the case study above.

\subsection{Identifying user questions}

In our example, SKIN was applied to a tender study with a clear client demand behind it, where the questions the simulation needs to answer were more or less pre-defined from the onset of the project. Enough time should, however, be dedicated to identifying and discussing the exact set of questions the stakeholders of the work want to see addressed.

We found that the best way to do this is applying an iterative process of communication between study team and clients, where stakeholders learn about the scope and applicability of the methods, and where researchers get acquainted with the problems policy makers have to solve and with the kind of decisions for which sound background information is needed. This iterative process should result in an agreed set of questions for the simulation, which will very often differ from the set proposed at the start of the study. In our example, a so-called "Steering Committee" was assigned to us by the European Commission consisting of policy makers and evaluation experts from DG INFSO.

There are various difficulties and limitations to overcome in identifying user questions. In the case of the DG INFSO study, although the questions under study were outlined in the Tender Specifications in great detail, this was a complicated negotiation process where the stakeholder group collectively reviewed the specific research objectives it wanted to investigate. In correspondence with the study team, the stakeholder group reflected on the exact nature and direction of their questions and reconsidered the original set of Tender Specifications, while negotiating among each other for an alternative set of questions. Stakeholders neither shared the same opinion about what questions should be in the final sample and how potential questions should be ranked in importance, nor shared the same hypotheses about questions in the final sample.

The specification of evaluative questions might be the first time stakeholders talk to each other and discuss their viewpoints.

In the INFSO-SKIN application, the following mechanism was used by the study team to identify user questions and proved to be valuable:

The written project specification is scanned by the client (in this case the Tender Specifications of DG INFSO) and the original set of questions is identified. A literature review and context analysis for each question (policy background, scope, meaning etc.) is conducted to inform the study team. In a meeting, the stakeholders present their views on written project specifications and their view on the context of questions. The study team informs the stakeholders on what the model is about, what it can and cannot do and discussions are held with stakeholders until the stakeholder group and the study team are "on the same page". The meeting is evaluated and the original set of questions is revised if necessary (probably an iterative process between study team and different stakeholders individually where study team acts as coordinator and mediator of the process).

A following meeting with stakeholders discusses the final set of questions and written consent on these questions is aimed at. Additionally, their hypotheses concerning potential answers and potential ways to address the questions are recognized.

The meeting is evaluated and experiments are developed that are able to operationalize the hypotheses and address the questions. Subsequently, feedback from stakeholders is requested, in order to assure 
consent that the experiments meet the questions and hypotheses. Finally, the meeting is evaluated and the experimental set-up concerning the final set of questions is refined.

This negotiation and discussion process are highly user-driven, interactive, and iterative. It requires communicative skills, patience, willingness to compromise on both sides, and motivation to make both ends meet - the formal world of modelers and the narrative world of policymaking in practice. The process is time-consuming. In our example, we needed about six months of a 12-months contract research study to get to satisfactory results on this first step.

\subsection{Getting their best: users need to provide data}

The study team will know best what types of empirical data are needed to inform the policy modeling. In SKIN, data availability is an important issue, because the findings need to be evidence-based and realistic. This is in the best interest of the stakeholders, who need to trust the findings. This will be the more likely to the extent that the simulated data resembles the empirical data known to the user (see section 2). However, the study team might discover that the desired data is not available, either because it does not exist or because it is not willingly released by the stakeholders or whoever holds it. In our example, the stakeholders were data collectors on a big scale themselves. The evaluation unit of DG INFSO employs a data collection group, which provides information about funded projects and organizations at a detailed level. Furthermore, the DG is used to providing data to the study teams they contract with for their evaluation projects. Consequently, we benefitted from having a large and clean database concerning all the matters the study team was interested in. However, it was still an issue to confirm the existence, quality and availability of the data and check for formats and database requirements. Even if the data is there in principal, enough time should be reserved for data management issues. The quality of the simulation in the eyes of the user will very much depend on the quality of the informing data and the quality of the model calibration.

What would have been the more common process if the study team had not struck lucky as in our example? In other SKIN applications, the following mechanism was used by the study team and proved to be valuable (the ones with asterisks apply to our INFSO-SKIN example as well):

The study team identifies the rough type of data which is required for the study from the project specifications and estimates the financial resources for data access in the proposal of the project (this can sometimes happen in interaction with the funding body). After a second meeting with stakeholders (see section 3.1), relevant data concerning variables to answer study questions and address/test hypotheses of section 3.1 is identified*. The study team then communicates exact data requirements to those stakeholders who are experts on their own empirical data environment and reviews existing data bases including the ones stakeholders might hold or can get access to*. Stakeholders meet with the team to discuss data issues and to help them understand and agree on the scope and limitations of data access*. Maybe further data is required by stakeholders and has to be collected. As final step, once again stakeholders and study team meet to discuss the final database, while afterwards the meeting is evaluated and data-to-model procedures are developed*.

\section{MODEL DISCUSSION}

The stakeholder group set the agenda of evaluative policy questions along with their needs and goals from a user perspective, which was further refined during the project in interaction with domain scientists and modelers. Stakeholders, domain experts and modelers agreed on and engaged in a moderated co-creation / co-design process (Voorberg et al. 2015) for ensuring the relevance and utility of the model for stakeholders, which included useful interface design and visualizations. 


\subsection{Interacting with users to check the validity of simulation results}

The stakeholders put considerable demands on the study team concerning understanding and trusting the simulation findings. The first and most important is that the clients want to understand the model. To trust results means to trust the process that produced them.

Here, the advantage of the adapted SKIN model is that it relies on a narrative that tells the story of the users' every-day world of decision-making (see section 2). In the SKIN model, a good example for "reality" requirements is the necessity to model the knowledge and behavior of agents. Blackboxing knowledge of agents or creating merely reactive simple agents would not have been an option, because stakeholders do not think the world works that way.

The SKIN model is based on empirical quantitative and qualitative research in innovation economics, sociology, science and technology studies, and business studies. Agents and behaviors are informed by what we know about them; the model is calibrated by data from this research. We found that there is a big advantage in having a model where stakeholders can recognize the relevant features they see at work in their social contexts. In setting up and adapting the model to study needs, stakeholders can actively intervene and ask for additional agent characteristics or behavioral rules; they can refine the model and enhance areas where they have information on the underlying processes.

However, here again, we encountered the diversity of stakeholder preferences. Different members of the DG INFSO Steering Committee opted for different changes and modifications of the model. Some were manageable within given time constraints and financial resources; some would have outlived the duration of the project if realized. The final course of action for adapting the model to study needs was the result of discussions between stakeholders about model credibility and increasing complexity and of discussions between stakeholders and the study team concerning feasibility and reducing complexity.

Once the stakeholders were familiar with the features of the model and had contributed to its adaptation to study requirements, there was an initial willingness to trust model findings. This was strengthened by letting the model reproduce FP7 data as the baseline scenario that all policy experiments would be benchmarked against. If the networks created by real life and those created by the agent-based model correspond closely, the simulation experiments can be characterized as 'history-friendly' experiments (cf. Malerba 2001), which reproduce the empirical data and cover the decisive mechanisms and resulting dynamics of the real networks.

\subsection{Visualization for understanding}

In presenting the results of the INFSO-SKIN study, however, it became clear that there were, again, caveats coming from the user community. The policy analysts did not want to look at a multitude of tables and scan through simulation results for interesting parameters; nor did they expect to watch the running model producing its results, because a typical run lasted 48 hours.

Presenting results in an appealing and convincing way required visualizations and interactive methods where users could intuitively understand what they see, had access to more detailed information if wanted, e.g. in a hyperlink structure, and could decide themselves in which format, in which order and in which detail they want to go through findings. This part of the process still needs further work: new visualization and interactive technologies can help to make simulation results more accessible to stakeholders.

\subsection{Discussing scope and limitations}

This leads to the last issue to be discussed. What happens after the credibility of simulation results is established? In the INFSO-SKIN study, the objective was policy advice for Horizon 2020. The stakeholders wanted the study team to communicate the results as "recommendations" rather than as 
"findings". They required a so-called "Utility Summary" that included statements, justified by the results of the study, about what they should do in their policy domain.

Here, the study team proved to be hesitant - not due to a lack of confidence in their model, but due to a recognition of its predictive limitations and a reluctance to formulate normative statements, which were seen as a matter of political opinion and not a responsibility of a scientific advisor.

The negotiation of the wording in the Utility Summary was another instance of an intense dialogue between stakeholders and study team. Nevertheless, the extent to which the results influenced or were somehow useful in the actual political process of finalizing Horizon 2020 policies was not part of the stakeholder feedback after the study ended and is still not known to us. The feedback consisted merely of a formal approval that we had fulfilled the project contract.

\subsection{Summary of workflow}

Derived from the lessons learned from the INFSO-SKIN study, we suggest a five-step process as a framework for co-designing social simulation models for policy advice. In the first step, the stakeholders' questions are identified through an iterative process that is likely to be work-intensive and should result in an agreed set of questions for the simulation model. In the second step, data requirements are to be identified in close cooperation with stakeholders and a discussion on a final database should be held. This will lead to the development of data-to-model procedures. In the third step, researchers and stakeholders together perform a validity check and make changes to agent characteristics or behaviors if necessary. This establishes trust in the validity and thereby in the results of the model. Visualizations are created in the fourth step in order to create accessibility and promote the understanding of the model among stakeholders. The fifth and last step is a discussion between researchers and stakeholders on the scope and limitations of the model. Clear communication on the simulation results and their implications is essential. 


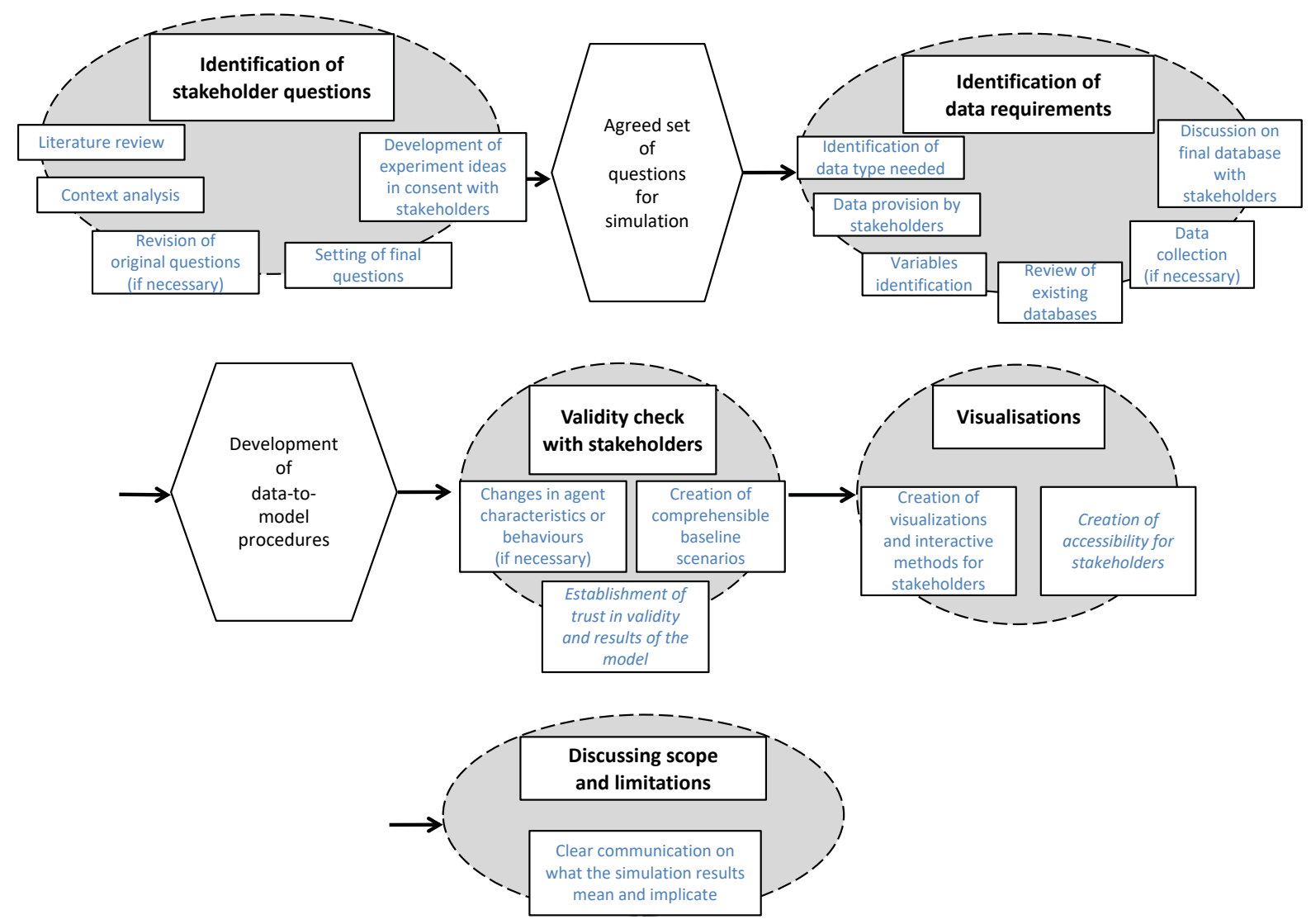

Figure 3: Flowchart of co-design workflow. The flowchart depicts the five building blocks that have been identified in the case study to be relevant steps in the co-design of social simulation models for policy advice.

\section{ACKNOWLEDGMENTS}

The authors appreciate the highly valuable and constructive comments by the assigned reviewers and thank these colleagues for contributing their time in order to improve this paper.

\section{REFERENCES}

Aodha, L., and B. Edmonds. 2017. "Some pitfalls to beware when applying models to issues of policy relevance”. In Simulating Social Complexity - a Handbook, 2nd edition, edited by B. Edmonds and R. Meyer, pp. 801-822. Heidelberg and New York, Springer.

Ahrweiler, P., N. Gilbert, and A. Pyka. eds. 2016. Joining Complexity Science and Social Simulation for Innovation Policy. Agent-based Modelling using the SKIN Platform. London, Cambridge Scholars Publishing.

Ahrweiler, P., M. Schilperoord, A. Pyka and N. Gilbert. 2015. "Modelling Research Policy - Ex-Ante Evaluation of complex Policy Instruments". Journal of Artificial Societies and Social Simulation $18 / 4 / 5$.

Ahrweiler, P. 2010. "Innovation in complex social systems. An introduction". In Innovation in complex social systems, edited by P. Ahrweiler, pp. 7-13. London and New York, Routledge. 
Barreteau, O., F. Bousquet, M. Étienne, V. Souchère, and P. d'Aquino. 2013. "Companion Modelling: A Method of Adaptive and Participatory Research". In Companion Modelling. A Participatory Approach to Support Sustainable Development, edited by M. Etienne, pp. 13-40. Heidelberg and New York, Springer.

Barreteau, O. et al. 2003. "Our Companion Modelling Approach". Journal of Artificial Societies and Social Simulation 6/1.

Berthet, E.T.A., C. Barnaud, N. Girard, J. Labatut, and G. Martin. 2016. "How to foster agroecological innovations? A comparison of participatory design methods". Journal of Environmental Planning and Management vol. 59/2, pp. 280-301.

Bouamrane, M., M. Spierenburg, A. Agrawal, A. Boureima, M.-C. Cormier-Salem, M. Etienne, C. Le Page, H. Levrel, and R. Mathevet. 2016. "Stakeholder engagement and biodiversity conservation challenges in social-ecological systems: some insights from biosphere reserves in western Africa and France". Ecology and Society 21/4.

Cacari-Stone, L., N. Wallerstein, A. P. Garcia, and M. Minkler. 2014. "The Promise of community-based participatory Research for Health Equity: a conceptual Model for bridging Evidence with Policy". American Journal for Public Health e1-e9.

Etienne, M. (ed.). 2013. Companion Modelling. A Participatory Approach to Support Sustainable Development. Heidelberg and New York, Springer.

Etzkowitz, H. and L. Leydesdorff. 1997. "A triple helix of university-industry-government relations". In Universities and the global knowledge economy. A triple helix of university-industry-government relations, edited by H. Etzkowitz and L. Leydesdorff, pp. 155-162. London and Washington, Pinter.

Geyer, R. and P. Cairney. eds. 2015. Handbook on Complexity and Public Policy. Cheltenham, Edward Elgar.

Gibbons, M., C. Limoges, H. Nowotny, S. Schwartzman, P. Scott and M. Trow. 1994. The new Production of Knowledge. London, Pinter.

Gilbert, N., P. Ahrweiler, P. Barbrook-Johnson, K. Narasimhan, and H. Wilkinson. 2018. "Computational Modelling of Public Policy: Reflections on Practice". Journal of Artificial Societies and Social Simulation 21/1/14.

Gilbert, N., P. Ahrweiler, and A. Pyka (eds.). 2014. Simulating Knowledge Dynamics in Innovation Networks. Heidelberg and New York, Springer.

Greenhalgh, T. et al. 2016. "Achieving Research Impact Through Co-creation in Community- Based Health Services: Literature Review and Case Study". Milbank Quarterly vol. 94/2, pp. 392-429.

Jager, W. and B. Edmonds. 2015. "Policy Making and Modelling in a Complex World". In Policy Practice and Digital Science, edited by M. Janssen, M. Wimmer and A. Deljoo, pp. 57-74. Heidelberg and New York, Springer.

Jones, N. A., P. Perez, T.G. Measham, G. J. Kelly, P. d'Aquino, K. A. Daniell, A. Dray, and N. Ferrand. 2009. "Evaluating Participatory Modeling: Developing a Framework for Cross-Case Analysis". Environmental Management vol. 44/6, pp. 1180-1195.

Lasecki, W. S. and J. P. Bigham. 2013. "Interactive Crowds: Real-Time Crowdsourcing and Crowd Agents". In Handbook of Human Computation edited by P. Michelucci, pp. 509-522. Heidelberg and New York, Springer.

Malerba, F., R. Nelson, L. Orsenigo, and S. Winter. 2001. "History-Friendly models: An overview of the case of the Computer Industry". Journal of Artificial Societies and Social Simulation 4(3), 6.

Mendoza, G. A. and R. Prabhu. 2006. "Participatory modeling and analysis for sustainable forest management: Overview of soft system dynamics models and applications". Forest Policy and Economics vol. 9/2, pp. 179-196. 
Ramaswamy, V. and K. Ozcan. 2014. The Co-Creation Paradigm. Redwood City, Stanford University Press.

Robles-Morua, A., K. . Halvorsen, A. S. Mayer, and E. R. Vivoni. 2014. "Exploring the application of participatory modeling approaches in the Sonora River Basin, Mexico". Environmental Modelling \& Software vol. 52, pp. 273-282.

Schilperoord, M. and P. Ahrweiler. 2014. "Towards a Prototype Policy Laboratory for Simulating Innovation Networks". In Simulating Knowledge Dynamics in Innovation Networks edited by N. Gilbert, P. Ahrweiler and A. Pyka, pp. 185-198. Heidelberg and New York, Springer.

Scholz, R., T. Nokkala, P. Ahrweiler, A. Pyka, and N. Gilbert. 2010. "The agent-based Nemo Model (SKEIN) - Simulating European Framework Programmes”. In Innovation in complex social Systems, edited by P. Ahrweiler, pp. 300-314. London, Routledge.

van Eeten, M. J. G., D. P. Loucks, and E. Roe. 2002. "Bringing actors together around large-scale water systems: Participatory modeling and other innovations". Knowledge, Technology \& Policy vol. 14/4, pp. 94-108.

Voorberg, W. H., V. J. J. M. Bekkers, and L. G. Tummers. 2015. "A Systematic Review of Co- Creation and Co-Production: Embarking on the social innovation journey". Public Management Review vol. 17/9, pp. 1333-1357.

\section{AUTHOR BIOGRAPHIES}

PETRA AHRWEILER is a Full Professor of Technology and Innovation Sociology / Social Simulation at Johannes Gutenberg University Mainz, Germany. She holds a PhD in Science and Technology Studies from Free University Berlin, Germany. Her research interests lie in innovation policy modeling, social simulation, and knowledge representation. Her email address is petra.ahrweiler@uni-mainz.de.

NIGEL GILBERT has a Distinguished Chair in Sociology at the University of Surrey, Guildford, UK and is Director of its Centre for Research in Social Simulation. He has a PhD in the Sociology of Scientific Knowledge from Cambridge University. His research interests include a wide range of areas at the interface between engineering, public policy and the social sciences. His email address is n.gilbert@surrey.ac.uk.

DEMIAN FRANK is a Research Fellow at the department of Technology and Innovation Sociology / Social Simulation of Johannes Gutenberg University Mainz, Germany. He holds an MA in social sciences and sports from Goethe University Frankfurt, Germany. His research interests lie in sociology of technology in general and artificial intelligence and its societal implications in particular. His email address is demifran@uni-mainz.de. 